Radial and Nonradial Pulsations as Probes of Stellar Physics

ASP Conference Series, Vol. 259, 2002

C. Aerts, T.R. Bedding, \& J. Christensen-Dalsgaard, eds.

\title{
Playing Music with Magnetic Fields
}

\author{
C. Foullon \\ CPA, K.U.Leuven, Belgium - University of St Andrews, Scotland ${ }^{1}$
}

\begin{abstract}
Through an increase in magnetic field strength, solar magnetic fields in the atmosphere or subsurface of the Sun can explain the frequency shifts observed on the time scale of the solar activity cycle. A separate study of the contribution of internal magnetic layers clarifies the relative importance of these effects. However, at the base of the convection zone, the cyclical change of orientation of magnetic field lines has a larger effect on global mode frequencies than an increase in field strength alone.
\end{abstract}

The standing waves in the Sun are by analogy like those in an organ pipe. Consider now, more specifically, a flute: near the driven end and in the vicinity of the open holes in the flute body, the pressure deviates substantially from that in an ideal pipe. Similarly, the gas pressure in the vicinity of a magnetic field deviates from that in a field-free Sun. By analogy to a flute, solar magnetic fields, as well as temperature changes, introduce pressure deviations that play a significant role in modulating the resonant frequencies of $p$-mode oscillations.

The effect of a thin horizontal buried magnetic layer on central $p$-mode frequencies is to encourage higher harmonics like a register hole in a flute (Foullon, 2001). In a Cartesian system $(x, y, z)$, a field-free polytropic medium is overlaid by a horizontal magnetic slab (with field lines aligned in the $x$-direction) of constant sound and Alfvén speeds (respectively $c_{c}$ and $v_{A}$ ), on top of a field-free polytrope; the adiabatically stratified field-free media are marginally stable to convective motions. The perturbations of the ideal linearised MHD equations are Fourier analysed proportionally to $\exp i\left(\omega t-k_{x} x-k_{y} y\right)$, where $\omega$ is the angular frequency of a mode of horizontal wavenumber $k_{h}=\left(k_{x}^{2}+k_{y}^{2}\right)^{1 / 2}$ (related to the degree $\ell$ through $k_{h}=L / \mathrm{R}_{\odot}$, for $L=\sqrt{\ell(\ell+1)}$ and solar radius $\left.\mathrm{R}_{\odot}\right)$.

Perpendicular to the magnetic field lines (with $k_{x}=0$ ), the characteristic speed is the fast magnetoacoustic speed $\left(c_{c}^{2}+v_{A}^{2}\right)^{1 / 2}$, which serves to increase the frequency of the modes propagating in that direction. By risking the analogy further, perpendicular wave propagation corresponds to playing the flute with a source of heat in front of the open hole, while parallel propagation $\left(k_{y}=0\right)$ indicates the ambient temperature at which the flute is played. For realistic condition parameters at the base of the convection zone (with maximum field strength $B_{o}=0.3 \mathrm{MG}$ ), the relative effect on $p$-mode frequencies of a buried magnetic layer of thickness $h=0.05 \mathrm{R}_{\odot}$ is much larger if the waves propagate perpendicular rather than parallel to the magnetic field lines (see Fig. 1).

\footnotetext{
${ }^{1}$ Present address: Royal Observatory of Belgium, email: Claire.Foullon@oma.be
} 

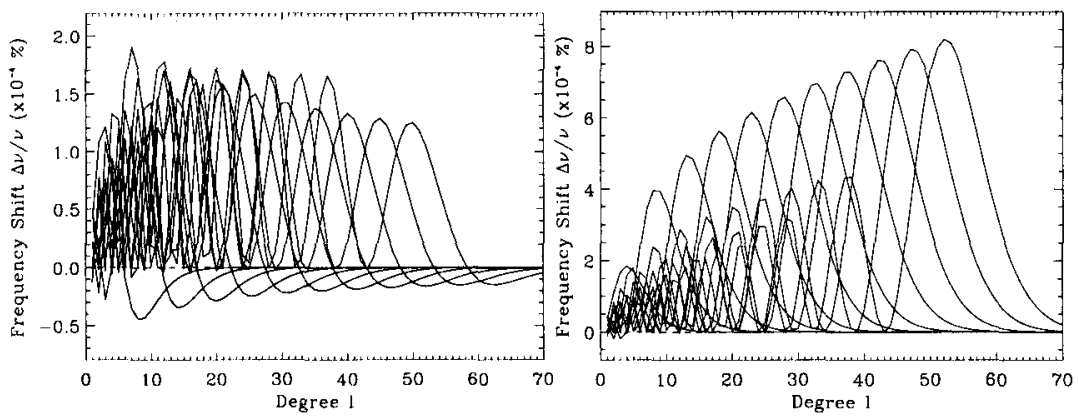

Figure 1. Percentages of frequency shifts versus degree $\ell$, due to (top) the sole effect of magnetic field in a buried layer below the convection zone, respectively for (top left) $k_{y}=0$ and (top right) $k_{x}=0$ and (right) the effect of perpendicular with respect to parallel propagation: analytical results are shown by lines joining shifts of same radial order $n$, from $n=1$ to $n=11$ (seen at higher $\ell$ ).

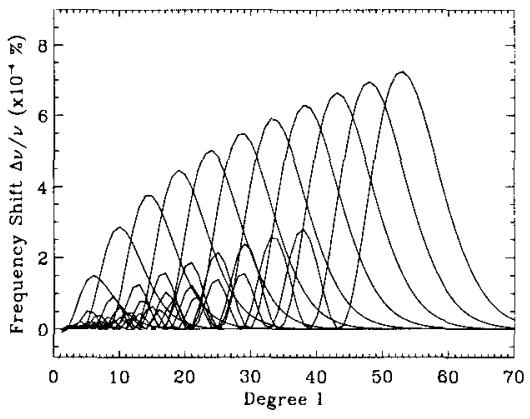

As the solar cycle rises, the $x$ and $y$-directions are presumed to flip by $90^{\circ}-$ the field lines passing from poloidal to toroidal at the base of the convection zone, see e.g. Dikpati \& Charbonneau (1999). Thus, instead of searching for an explanation through a variation in field strength, one may also interpret the observed shifts in global frequencies by a change in orientation of the magnetic field lines. At solar maximum, the 'global' waves (i.e. as if the Sun were not rotating) propagate perpendicular to a toroidal field; at solar minimum, they propagate parallel to a poloidal one. This change of configuration would produce maximum relative frequency shifts almost as large as the the sole effect of the field strength would have on perpendicular propagating waves (see Fig. 1).

However, the frequency cutoffs and large influence of the changing atmosphere prevent from comparing internal effects with observations. For the tachocline model with $k_{x}=0$, an effect as large as $\Delta \nu / \nu \sim 9 \times 10^{-4} \%$ at the peak shift frequency $3900 \mu \mathrm{Hz}$ is still negligible in comparison to the observations $\left(\Delta \nu / \nu \sim 2 \times 10^{-2} \%\right.$ over a solar cycle). Details will be provided in Foullon (2002, PhD Thesis, in preparation).

Acknowledgments. Special thanks to the European Research Training Network PLATON for the financial support (grant No. HPRN-CT-2000-00153).

\section{References}

Dikpati, M. \& Charbonneau, P. 1999, ApJ, 518, 508

Foullon, C. 2001, in INTAS Work. Proc., MHD Waves in Astrophysical Plasmas, ed. J.L. Ballester \& B. Roberts, in press 